\title{
A Health Systems Genogram for Improving Hospital Transitions to Primary Care
}

\author{
Max Zubatsky, $P b D^{*}$ and Jay Brieler, $M D$ \\ Department of Family and Community Medicine, \\ Saint Louis University, St Louis, Missouri \\ Ann Fam Med 2018;16:566. https://doi.org/10.1370/afm.2318.
}

\section{THE INNOVATION}

Integrated Behavioral Health (IBH) has become a standard of care that addresses gaps in the delivery of mental health services in this country. ${ }^{1}$ One challenge of IBH is finding common language that clinicians at multiple levels of training can share. ${ }^{2}$ We introduce the Health Systems Genogram, a clinical tool designed to improve coordination between clinicians in IBH settings, used to supplement follow-up care for patients transitioning from the hospital to an outpatient setting.

\section{WHO AND WHERE}

We are a university-based, Midwestern family medicine residency that trains residents at a community hospital and a Federally Qualified Health Center (FQHC). Additionally, Behavioral Health $(\mathrm{BH})$ graduate students at both Master's and PhD levels train in an integrated care model at both the $\mathrm{FQHC}$ and 3 other primary care clinics.

\section{HOW}

This assessment was created in response to: (1) the lack of a concrete tool around the parameters of communication between medical and $\mathrm{BH}$ clinicians at differing levels of training, and (2) a system-wide need to improve the transition from inpatient to outpatient primary care in underserved communities. Our initial aim was to utilize behavioral health graduate students to assess the systemic and social factors that impact patients' likelihood to follow through with outpatient appointments after discharge from the hospital. Patients within 48 hours of hospital discharge were targeted to receive a comprehensive health systems assessment by our BH learners.

Conducting a thorough family assessment in the hospital setting was often time-consuming, requiring clinicians to document this information in different places. With the development of the Health Systems Genogram, we combine the essential elements of the assessment into one straightforward instrument (Supplemental Figure 1, http://www.annfammed.org/ content/16/6/566/suppl/DC1). We have retained the traditional

Conflicts of interest: authors report none.

*Corresponding author: max.zubatsky@health.slu.edu 3-generation genogram, ${ }^{3}$ including medical and mental health conditions, family history, relationship patterns, cultural themes, and employment history. Additional sections gather information on appointment scheduling, medical management, transportation, and members of the family system involved in care. Connections are drawn between members in the genogram and responses around health management and decision making.

In this example, the patient provided both the $\mathrm{BH}$ provider and physician with family history and pertinent themes within generations. The main medical and mental health issues (ie, diabetes, depression, chronic obstruction pulmonary disease, and alcohol abuse) are listed in a legend on the left side of the genogram. This patient, who has complications with diabetes management and depression, communicates mostly with his mother and brother about his health care needs. The brother is also responsible for managing health care appointments, including transportation. The brother and/or mother would be important allies in care during the patient's initial follow-up appointment, and could potentially provide more information about medical and social issues present in the family system. Additionally, the team now has information about the main clinicians involved in the patient's plan of care when discharged.

When the assessment is completed, the genogram is presented in verbal and written form with the inpatient medical team, and scanned into the outpatient electronic health record for use by the outpatient primary care team. Prior to the patient's initial primary care follow-up appointment, the physician, $\mathrm{BH}$ provider, and diabetes educator conduct a previsit consultation to optimize the encounter. Using the Health Systems Genogram, clinicians can review the psychosocial and family systems contexts that were identified in the hospital at a greater depth than within the typical flow of outpatient practice.

\section{LEARNING}

We have conducted over 40 assessments in the hospital for patients transitioning to follow-up with their physician at our FQHC. The instrument has allowed for greater collaboration and communication among physicians, BH providers, and other health professionals. The assessments have also improved resident awareness of systemic factors in patient care, and have allowed transfer of significant amounts of information in a short time as clinicians have become familiar with the format.

The corresponding author information, supplemental figure, key words, submitted dates, funding support statement, and references are available at http:I/www.AnnFamMed.orgl content/16/6/566/suppl/DC1. 